\title{
RELAÇÃO ENTRE HIGIENE BUCAL E GENGIVITE DE UMA POPULAÇÃO EM SITUAÇÃO DE POBREZA
}

\section{RELATIONSHIP BETWEEN ORAL HYGIENE AND GUM DISEASE IN A POPULATION IN A SITUATION OF POVERTY}

\author{
Márcia Cançado Figueiredo ${ }^{1 *}$, Mariéle Darros Kunkel ${ }^{1}$, Kátia Valença Correia \\ Leandro da Silva ${ }^{2}$ \\ ${ }^{1}$ Universidade Federal do Rio Grande do Sul, Faculdade de Odontologia, \\ Departamento de Cirurgia e Ortopedia, Porto Alegre, Rio Grande do Sul, Brasil. ${ }^{2}$ \\ Universidade Federal do Rio Grande do Sul, Instituto de Biociências, Porto Alegre, \\ Rio Grande do Sul, Brasil \\ *Autor correspondente: Rua Ramiro Barcelos, 2492. Bom Fim, CEP 90520- 080 - Porto \\ Alegre, RS - Brasil - Caixa-postal: 90520080 Telefone: (51) 33085027 Fax: (51) 33375471. \\ E-mail:mcf1958@gmail.com
}

\section{RESUMO}

Avaliar as condições de saúde bucal dos moradores do bairro Augusta Meneguine, através dos índices de placa visível e sangramento gengival, relacionando-as com o perfil socioeconômico. Foi realizado um estudo do tipo transversal, observacional e analítico contendo uma amostra de 684 moradores de um bairro do município de Viamão, Rio Grande do Sul. Os dados foram coletados durante os mutirões interdisciplinares de saúde de março 2014 a julho de 2015. Os participantes responderam a um questionário contendo informações referentes às condições socioeconômicas, além de dados sobre a saúde geral e bucal. Foram avaliadas a presença de placa visível e sangramento gengival após a escovação dentária. Os dados foram armazenados no programa Microsoft Excel e analisados quantitativamente, com tabelamento em percentual, sendo apresentados em frequência relativa absoluta. A média de idade da população avaliada foi de 23,4 anos, havendo uma prevalência de mulheres na amostra. A maior parte das pessoas tinha escolaridade de $1^{\circ}$ grau incompleto. A renda familiar mais encontrada na população estudada foi de até 1 salário mínimo. Com relação à presença de placa, $71 \%$ apresentaram placa visível. Já quanto à presença de sangramento gengival, o resultado positivo igualmente prevaleceu na grandeza de 55\%. Através deste levantamento, foi possível identificar um perfil precário de saúde bucal e de nível sócio econômico dos moradores da Vila Augusta: baixas remuneração e escolaridade, apresentando altos índices de placa visível e sangramento gengival.

Palavras-chave: Doença periodontal. Saúde Bucal. Pobreza.

\begin{abstract}
It was aimed to assess oral health conditions of the residents of the Augusta Meneguine neighborhood through the indexes of visible plaque and gingival bleeding, relating them to the socio-economic profile. It was conducted a transverse observational and analytical study containing a sample of 684 residents of a subdivision of the city of Viamão, Rio Grande do Sul. Data were collected during the interdisciplinary master of health from march 2014 to july 2015. Participants answered to a questionnaire containing information regarding socioeconomic conditions, in addition to data on the general health and dental. We evaluated the presence of visible plaque and gingival bleeding after tooth brushing. Data was stored in Microsoft Excel and analyzed quantitatively, with pegging in percentage, being presented in absolute-relative frequency. The average age of the population evaluated was 23.4 years, with a prevalence of women in the sample. Most people had 1st school degree incomplete. Family income more found in the studied population was up to 1 minimum wage. About the presence of plaque,
\end{abstract}


$71 \%$ showed visible card. Already with regard to the presence of gingival bleeding, the positive result also prevailed in the greatness of 55\%. Through this survey, it was possible to identify a precarious dental health profile and socio economic level of the residents of the village Augusta: low pay and schooling, showing high levels of visible card, and gum bleeding.

Keywords: Periodontal Diseases. Oral Health. Poverty

\section{INTRODUÇÃO}

As doenças da cavidade bucal são evitáveis pelo emprego correto de medidas preventivas, mas pouco se conhece sobre os fatores que condicionam os indivíduos a assumirem ou não um comportamento preventivo. Os modelos tradicionais de atenção, em sua maioria, são baseados no pressuposto de que o comportamento é regido por determinantes internos no nível do indivíduo como, por exemplo, percepção, crença, atitude ou intenção. Contudo, são importantes as condições socioeconômicas e ambientais, que forçosamente condicionam o comportamento de cada pessoa. (SHEIHAM,2000).

Para Santos, et al. em 2004, a taxa de analfabetismo, isoladamente, não explicita as carências educacionais de uma população estudada, é necessário perceber a dificuldade de acesso ao ensino médio e superior, para compreender a exclusão dessa população. Neste estudo, ainda se verificou que $100 \%$ das crianças com seis ou mais dentes cariados eram cuidadas por indivíduos com baixa escolaridade ( $1^{\circ}$ grau incompleto ou analfabetos), enquanto todas as crianças cuidadas por indivíduos que completaram no mínimo o $1^{\circ}$ grau possuíam um índice ceo-d (dentes decíduos cariados, com extração indicada e obturados), abaixo de cinco.

Estudo de Dockhorn e Pretto comprovaram a importância da educação, em particular da mãe, na saúde bucal das crianças. Isso se dá tanto pelo reflexo da própria condição de saúde bucal da mãe, como da implementação de cuidados de alimentação e higienização decorrentes de maior grau de informação da mesma.

Acrescentando, Aquino e Philippi, em 2002, relataram que o consumo de açúcar é maior entre as crianças de menor renda. Já Figueiredo et al., em 2014, constatou que existe uma associação positiva entre o consumo de sacarose com o aparecimento da cárie em crianças, onde foi encontrada uma associação significante entre as variáveis de renda e frequência de mais ingestões de açúcar entre as refeições, nas quais as famílias com renda salarial mais elevada tiveram um maior consumo de açúcar entre as refeições comparadas àquelas com menor renda, cuja frequência de ingestão de açúcar foi significativamente menor e, entre as variáveis "tipo de casa" e "frequência de higiene bucal realizada", a qual era feita com maior frequência ao dia pelos moradores de casa do tipo alvenaria.

Oppermann e Rösing descreveram a gengivite, como uma das doenças bucais de maior prevalência, sendo encontrada em todas as idades, desde que a placa se acumule por um certo período de tempo. Gesser, Peres e Marcenes verificaram em 2001, uma alta prevalência de sangramento e cálculo dentário em indivíduos com piores condições socioeconômicas. A análise estatística demonstrou associação significativa entre sangramento gengival e todas as variáveis socioeconômicas estudadas, chegando à conclusão de que quanto maior a escolaridade e a renda familiar, menor a prevalência de sangramento gengival.

Na mesma lógica, Maltz e Barbachan, em 2001, observaram associações locais entre o índice de sangramento gengival ISG $\leq 10 \%$ de escolares com pais de nível superior completo e o ISG $\leq 30 \%$ para pais de nível educacional até primeiro grau incompleto. Neves et al., em 2013, também concluíram que indivíduos com piores índice de placa e de sondagem periodontal estiveram associados à piores indicadores sociodemográficos e psicocomportamentais.

Um resultado extremamente interessante foi percebido quando Neves, Passos e Oliveira examinaram 176 escolares de uma instituição da rede pública de ensino da cidade de João Pessoa. As crianças examinadas apresentaram elevada prevalência de gengivite na forma leve, apesar de terem relatado uma alta frequência de escovação. Foi observada uma diferença 
estatisticamente significante para os valores do índice de sangramento gengival entre os indivíduos que relataram escovar seus dentes uma vez ao dia com os que escovavam duas vezes ao dia ou também entre os que escovavam três vezes ou mais ao dia.

Por outro lado, Maltz e Barbachan afirmaram que essa relação de baixo nível socioeconômico e alta incidência de gengivite pode ser atribuída ao fato de as pessoas de menor poder aquisitivo terem menos acesso à assistência odontológica, menor grau de instrução, inclusive as relacionadas aos cuidados com higiene bucal, o que pode acarretar menos preocupação com a saúde e o bem-estar. Complementando, Ruffino afirmou que a distribuição de recursos, ainda, é inversamente proporcional às necessidades da população, uma vez que a desigualdade da distribuição dos serviços não corresponde às necessidades das mesmas.

Devido a estas constatações, o presente trabalho teve como objetivo avaliar as condições de saúde bucal, através dos índices de placa visível e sangramento gengival, e o perfil socioeconômico dos moradores do bairro Augusta Meneguine, do município de Viamão, RS.

\section{MATERIAIS E MÉTODOS}

Este trabalho é do tipo transversal, observacional e analítico. Ele foi realizado na Vila Augusta Meneguine, no município de Viamão, Rio Grande do Sul.

O estudo abrangeu uma amostra por conveniência, constituída de 684 moradores da referida Vila que assinaram um termo de consentimento livre esclarecido. Foram excluídas aquelas pessoas que não consentiram com o termo da pesquisa.

Os dados foram coletados durante atividades de extensões interdisciplinares de saúde ali realizados aos sábados, dos meses de março 2014 a julho de 2015.

Todos também responderam a um questionário padronizado que buscava informações referentes às condições socioeconômicas do chefe da família, a escolaridade, além de dados sobre a saúde geral e saúde bucal (orientação de higiene bucal, frequência de higiene bucal, frequência de ingestão de açúcar).

As condições de saúde bucal desses moradores foram avaliadas utilizando-se os critérios de presença ou ausência de placa visível (ISG) e sangramento gengival (IPV). Esses exames bucais foram realizados por examinadores treinados mediante luz artificial nos ambientes mais claros das residências, sendo iniciados pela verificação da presença de placa visível, seguida da escovação supervisionada e da observação de sangramento gengival (Figura 1).

Os dados coletados foram armazenados no programa Microsoft Excel 2010 e analisados

Figura 1 - Escovação dentária sendo realizada para posterior realização do exame clínico.

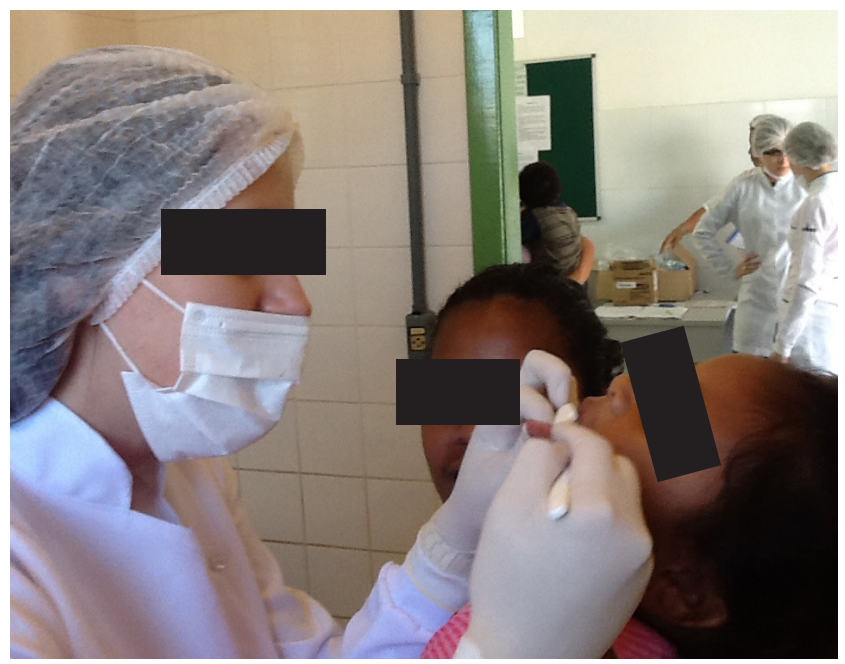

Fonte: Márcia Cançado Figueiredo

estatisticamente pelo Teste Qui-quadrado e para comparação entre as variáveis utilizou-se o Teste Exato de Fisher. O presente trabalho foi aprovado pelo Comitê de Ética em Pesquisa da SMS de Porto Alegre sob o número 669, processo: 001.032690.11.8, datado de 09/08/2011.

\section{RESULTADOS}

Do total da amostra de 684 indivíduos com idade média 23,4 anos de idade, dos quais $36,55 \%(n=250)$ são homens com uma média de idade de 20,04 anos, e $63,45 \%(n=434)$ são mulheres com idade média de 25,4 anos. Conforme a Tabela 1.

Tabela 1 - Média de idade, número de indivíduos e percentual na população

\begin{tabular}{c|c|c}
\hline & Homens & Mulheres \\
\hline Média de idade & 20,04 & 25,4 \\
\hline $\mathbf{N}^{\circ}$ de indivíduos & 250 & 434 \\
\hline \% na população & 36,55 & 63,45 \\
\hline
\end{tabular}

Fonte: Mariéle Darros Kunkel

No levantamento socioeconômico, analisaram-se os índices de escolaridade e renda mensal. Verificouse que $62,2 \%(n=426)$ não completaram o Ensino Fundamental (Tabela 2). O analfabetismo estava presente em 13,4\% (n=92) da população geral. Em relação 
ao item renda mensal, 35,5\% $(\mathrm{n}=243)$ dos entrevistados relataram uma variação de renda de um a dois salários mínimos (SM). Em primeiro lugar, destaca-se a renda familiar de até um salário mínimo por mês, presente em $54,5 \%(n=373)$ da amostra total (Tabela 3).

Tabela 2 - Escolaridade da população

\begin{tabular}{c|c}
\hline & População Total \\
\hline Analfabetos & $13,4 \%(92)$ \\
\hline $\mathbf{1}^{\mathbf{0}}$ Incompleto & $62,2 \%(426)$ \\
\hline $\mathbf{1}^{\mathbf{0}}$ Completo & $6 \%(42)$ \\
\hline $\mathbf{2}^{\mathbf{0}}$ Incompleto & $11,2 \%(77)$ \\
\hline $\mathbf{2}^{\mathbf{o}}$ Completo & $6 \%(42)$ \\
\hline $\mathbf{3}^{\mathbf{O}}$ Incompleto & $0,14 \%(1)$ \\
\hline $\mathbf{3}^{\mathbf{0}}$ Completo & $0,6 \%(4)$ \\
\hline
\end{tabular}

Tabela 3 - Renda mensal da população

\begin{tabular}{c|c}
\hline & População Total \\
\hline Sem Renda & $0,73 \%(5)$ \\
\hline Até 1 SM & $54,5 \%(373)$ \\
\hline De 1 a 2 SM & $35,5 \%(243)$ \\
\hline De 3 a 5 SM & $9 \%(62)$ \\
\hline Mais de 5 SM & $0,14 \%(1)$ \\
\hline
\end{tabular}

Fonte: Mariéle Darros Kunkel

Fonte: Mariéle Darros Kunkel

Com relação à presença de placa, $71 \%(\mathrm{n}=485)$ apresentaram placa visível. Já quanto à presença de sangramento gengival, o resultado positivo igualmente prevaleceu na grandeza de 55\% $(n=377)$. Quando questionados sobre a frequência de escovação, 3,65\% (25) afirmaram não escovar os dentes; 29,09\% (n=199) relataram escovar até uma vez ao dia; $55,70 \%(\mathrm{n}=381)$ relataram escovar de duas a três vezes ao dia; e $11,55 \%(\mathrm{n}=79)$ escovavam quatro vezes ou mais ao dia. Em relação à orientação de higiene bucal, $67 \%(\mathrm{n}=457)$ relataram nunca haver recebido qualquer tipo de orientação ao longo de sua vida. Os entrevistados foram questionados em relação à frequência de consumo de açúcar entre as refeições, conforme demonstrado na Tabela 5.

Tabela 4 - Frequência de escovação, placa visível, sangramento gengival

\begin{tabular}{c|c|c}
\hline Frequência de escovação diária & Placa Visível & Sangramento gengival \\
\hline Nenhuma X ao dia $3,65 \%(\mathrm{n}=25)$ & $68,00 \%(17)$ & $52,00 \%(13)$ \\
\hline Até 1X ao dia 29,09\% $(\mathrm{n}=199)$ & $69,34 \%(138)$ & $48,74 \%(97)$ \\
\hline De 2 a 3 X ao dia 55,70\% $(\mathrm{n}=381)$ & $72,70 \%(277)$ & $58,79 \%(224)$ \\
\hline 4X ou +X ao dia $11,55 \%(\mathrm{n}=79)$ & $64,55 \%(51)$ & $53,16 \%(42)$ \\
\hline
\end{tabular}

Fonte: Mariéle Darros Kunkel

Tabela 5 - Frequência de ingestão de açúcar entre as refeições

\begin{tabular}{c|c}
\hline Ingestão de açúcar entre refeições & População Total \\
\hline Nenhuma vez & $1 \%(7)$ \\
\hline $1 \mathrm{X}$ & $10,3 \%(71)$ \\
\hline $2 \mathrm{X}$ & $23,8 \%(163)$ \\
\hline $3 \mathrm{X}$ & $30 \%(206)$ \\
\hline Mais de 3 X & $34,3 \%(235)$ \\
\hline Mamadeira Noturna & $0,3 \%(2)$ \\
\hline
\end{tabular}

Fonte: Mariéle Darros Kunkel

\section{DISCUSSÃO}

A população trabalhada foi caracterizada como jovem (média de 23,4 anos de idade) e, por viver abaixo da linha da pobreza, uma vez que as famílias sobreviviam com menos de um salário mínimo por mês e em sua maioria eram compostas por 3 a 5 moradores por residência em situação precária. Isto reflete a realidade vivida também por 16,2 milhões de pessoas que se encontram em pobreza extrema de acordo com dados do Censo 2010, do Instituto Brasileiro de Geografia e Estatística (IBGE, 2011). Um entre cada dez brasileiros vive em condições de extrema pobreza, sendo que na região Nordeste é concentrado a maior parte dos extremamente pobres - 9,61 milhões de pessoas ou $59,1 \%$. Destes, a maior parcela $(56,4 \%)$ vive no campo, enquanto $43,6 \%$ estão em áreas urbanas. A região Sudeste tem 2,72 milhões de brasileiros em situação de miséria, seguido pelo Norte, com 2,65 milhões, pelo Sul (715,96 mil), e o Centro Oeste (557,44 mil)(Tabela 1 e 3) (IBGE,2011).

Deste modo, a realidade encontrada na Vila Augusta reflete a situação dos muitos dos achados deste estudo, como, por exemplo, a relação proporcional da renda dos moradores com sua frequência de ingestão 
de açúcar entre refeições. Dados estes que corroboraram com os de Aquino e Philippi, que verificaram que o consumo de açúcar na classe de menores rendimentos foi superior ao observado na classe de maiores rendimentos, sustentando a hipótese de que o nível socioeconômico influencia a preferência pelo açúcar. Estes dados não foram corroborados por Figueiredo et al. em 2014, que observaram uma frequência de mais ingestões de açúcar entre as refeições em famílias com renda salarial mais elevada (Tabela 5).

Na literatura é consagrada a associação do nível sócio econômico com os hábitos de higiene bucal. Os resultados de Figueiredo et al. no ano de 2014 revelaram uma associação significante entre a estrutura da residência (alvenaria, madeira ou mista) e a frequência de higiene bucal por dia realizada pelos moradores de uma vila. As famílias que residiam em domicílios do tipo alvenaria apresentaram uma maior frequência de higiene bucal do que aquelas cujas moradias eram feitas de madeira ou mesmo mista. Pode-se dizer que o espaço físico onde a pessoa reside pode influir em sua saúde bucal.

Conforme Moreira, Nico e Tomita (2007, p. 278-9), "deve-se reconhecer uma reprodução de expressão espacial das condições de vida e saúde da população na medida em que o local de moradia do indivíduo reflete sua própria condição socioeconômica. Dessa maneira, o território, como reflexo das condições econômicas de seus habitantes e sujeito às iniquidades sociais e políticas, poderia influenciar negativamente as condições de vida de seus ocupantes. Percebe-se, então, que o território passa a gerar (e gerir) a pobreza de forma sustentável”.

Os resultados também apontaram altos índices de presença de placa visível e sangramento gengival, sendo ambos encontrados em mais da metade da amostra $(71 \%$ e $55 \%$, respectivamente). Esses resultados reiteram os achados de Gesser, Peres e Marcenes, que verificaram alta prevalência de sangramento e cálculo dentário em indivíduos com piores condições socioeconômicas em 2001, em uma amostra populacional de 286 jovens de 18 anos de idade do município de Florianópolis, os quais revelaram que $86 \%$ dos indivíduos apresentaram sangramento à sondagem. Considerando que a população em estudo foi composta majoritariamente por indivíduos de baixa renda e escolaridade, justifica-se a alta prevalência desses índices periodontais.
Acompanhando a mesma lógica descrita acima, Maltz, Barbachan e Neves, et al., afirmaram que os altos índices de placa e sangramento gengival estão associados à piores indicadores sociodemográficos e psicocomportamentais. Cabe ressaltar que aqui a maioria da população estudada tinha apenas o $1^{\circ}$ grau incompleto (Tabela 2).

As condições sociais mais baixas, portanto, não resultaram em hábitos de higiene bucal que se possa chamar de inadequados (ainda que os resultados os demonstrem ineficazes), sugerindo que a alta frequência de escovação não necessariamente implica em melhor limpeza. Pode-se pensar, no entanto, que a discrepância entre auto relato e exame clínico refletiria uma alta valorização do cuidado com a saúde por parte da população, o que colocaria em dúvida a confiabilidade das respostas aos questionários aplicados, conforme discutido por Figueiredo et al., em 2011.

Considerando que, independente da frequência de escovação, os índices de placa visível e sangramento gengival permaneceram com uma porcentagem próxima entre si, torna-se compreensível a especulação descrita acima. Parece claro que, supondo-se verdadeiras as respostas ao questionário, os maus índices de higiene bucal podem derivar de técnica e/ou instrumentos inadequados (Tabela 4).

Realmente, foi possível perceber a necessidade de uma adaptação nas políticas públicas de saúde voltadas para a atenção básica, com base em educação em saúde, com distribuição de escovas dentárias, fio dental e pasta de dente. Exemplificando, segundo o Instituto Brasileiro de Geografia e Estatística (IBGE), na Pesquisa Nacional de Saúde, que conta com dados de 2013, o uso de escova de dente, pasta de dente e fio dental para a higiene bucal é feito somente por $53 \%$ dos brasileiros. Ainda nesta pesquisa, os homens usam menos os artigos de higiene bucal que as mulheres. Enquanto $57,1 \%$ delas declararam os cuidados com a boca, $48,4 \%$ dos homens afirmaram usar escova, pasta e fio de dental.

Finalizando, apesar da progressiva democratização do acesso à saúde para a população e os crescentes programas de combate à miséria, as condições de pobreza extrema prevalecem nos centros urbanos e, conforme afirmado anteriormente, contribuem substancialmente para a prevalência de doenças bucais. Fica um indicativo de que é necessário adaptar as políticas públicas de saúde bucal às características de cada 
região, se for um município de pequeno porte como o de Viamão, o acesso será diferenciado dos de grande porte. Também é importante que a formação do profissional de saúde esteja mais adaptada para atender a realidade vivida pela população brasileira através das reformas curriculares que contemplam os princípios do cuidado humanizado, integral e descentralizado à população.

Assim, as atividades de extensão interdisciplinares em saúde, semelhante às adotadas no desenvolvimento deste trabalho, para promover saúde através do atendimento a uma população desassistida como a da Vila Augusta, devem ser adotadas pelas universidades de forma que haja uma contribuição para a sociedade e, concomitantemente, prepare o aluno de graduação para uma formação profissional inserida na realidade do país.

\section{CONCLUSÃO}

Através deste levantamento foi possível identificar na população dos moradores da Vila Augusta: altos índices de placa visível, e sangramento gengival.

\section{AGRADECIMENTOS}

Agradecemos a Dra. Dilene Dumke, cirurgiã-dentista da Secretaria Municipal de Saúde do município de Viamão, a Auxiliar em Saúde Bucal (ASB) Simone da Rosa e toda equipe da Extensão Universitária: "Ação Interdisciplinar em Saúde" pelo apoio constante e informações cedidas durante a realização deste trabalho.

\section{REFERÊNCIAS}

Aquino, R de C; Philippi, ST. Consumo infantil de alimentos industrializados e renda familiar na cidade de São Paulo. Rev Saúde Publica,v.36,n.6,p.655-60, dec.2002.

Brasil. Ministério do Planejamento, Orçamento e Gestão. Instituto Brasileiro de Geografia e Estatística. Percepção do estado de saúde, estilo de vida e doenças crônicas: Brasil grandes regiões e unidades da Federação. Pesquisa Nacional de Saúde 2013. Rio de Janeiro: 2014.p.0-181.

Brasil. Ministério da Saúde. Secretaria de Atenção à Saúde. Departamento de Atenção Básica. Guia de recomendações para uso de fluoretos no Brasil. Série A. Normas e manuais técnicos. Brasília: 2009.p.0-58.

Dockhorn, DMC ; Pretto, SM. Escolaridade, condições socioeconômicas e saúde bucal: relação entre mães e filhos. Rev. odonto ciência, v.16, n.32, p.42-59, jan/abr. 2001.
Figueiredo, MC et al. Saúde bucal de moradores de um bairro pobre de Xangri-lá, RS, Brasil. ConScientiae Saúde,v.10,p.2,n.177-83. 2011

Figueiredo, MC et al. Descrição do padrão alimentar e da saúde bucal de crianças do sul do Brasil. RFOUFP,v.19,n.3,p.329-36, set/dez.2014.

Fraiz, FC ; Walter, LR de F. Study of the factors associated with dental caries in children who received early dental care. Pesquisa Odontol Bras, v.5, n.3, p.201-7, jul/set. 2001

Gesser, HC; Peres, MA; Marcenes, W. Condições gengivais e periodontais associadas a fatores socioeconômicos. Rev Saúde Pública, v.35, n.3, p.289-93. 2001.

Instituto Brasileiro de Geografia e Estatística. Indicadores sociais municipais: uma análise dos resultados do universo do censo demográfico, IBGE: 2010. Rio de Janeiro. p. 0-149. 2011.

Kowash, MB, et al. Effectiveness on oral health of a long term health education programme for mothers with young children, Br Dent J, v.188,n.4,p.201-5,feb.2000.

Maltz,M; Barbachan,SB. Relação entre cárie, gengivite e fluorose e nível socioeconômicos em escolares. Rev Saúde Publica, v.35,n.2,p.170-6, apr.2001.

Moreira, SR; Nico, SL; Tomita, NE. A relação entre o espaço e a saúde bucal coletiva: por uma epidemiologia georreferenciada. Ciênc Saúde Coletiva,v.12,n.1,p.275-84. 2007

Neves, AM; Passos, IA; Oliveira, AFB. Estudo da prevalência e severidade de gengivite em população de baixo nível socioeconômico. Odontol Clín Cient,v.9,n.1,p.65-71, jan/mar.2010.

Neves, PCB, et al. Variáveis sociodemográficas e psico comportamentais associadas à gengivite e à pobre higiene bucal em pacientes de uma unidade de saúde da família. Braz J Periodontol,v.23,n.2,p.16-24, jun. 2013

Oppermann, RV; Rösing, CK. Prevenção e tratamento das doenças periodontais. In: KRIGER L, Bezerra ACB (Org.). ABOPREV: promoção de saúde bucal. São Paulo: Artes Médicas, 1997. 300 p.

Ruffino, NA. Qualidade de vida: compromisso histórico da epidemiologia. In: Congresso da ABRASCO,2., Belo Horizonte, 1993 Anais do $2^{\circ}$ Congresso Brasileiro de Epidemiologia; Belo Horizonte: ABRASCO, 1993.p.11-8.

Santos, MF. et al. Relação entre doença cárie e gengivite e condições socioeconômicas dos usuários da creche comunitária centro infantil Murialdo. Biol. Saúde, v.18, n.1, p113-26, jan/jun. 2004.

Sheiham, A. A determinação de necessidades de tratamento odontológico: uma abordagem social. In: Pinto VG. Saúde bucal coletiva. São Paulo: Santos, 2000. 270p. 